\title{
Effect of Rehabilitation Training on the Recovery of Hemiplegic Limb in Patients with Cerebral Infarction
}

\author{
L. MA*, Q. DENG, L. DONG1, YAN TANG AND L. FAN \\ Department of Rehabilitation Medicine, Taizhou Hospital of Zhejiang Province, Taizhou Enze Medical Center (Group), Linhai \\ 317000, Zhejiang Province, ${ }^{1}$ Dispensary, Linhai Hospital of Traditional Chinese Medicine, Linhai 317000, Zhejiang Province, \\ China
}

Ma et al.: Effect of rehabilitation training on limb recovery of patients with cerebral infarction hemiplegia

\begin{abstract}
The purpose of this study is to explore the effect of rehabilitation training on limb recovery of patients with cerebral infarction hemiplegia and the improvement of daily life activities of patients. In this study, 46 patients with cerebral infarction hemiplegia are selected as the research subjects. All of them receive rehabilitation training within 0 to $24 \mathrm{~h}$ after the acute phase of cerebral infarction and the patient's vital signs are stable. After rehabilitation training for $2 \mathrm{w}$ and $4 \mathrm{w}$, the motor function and daily life activity of hemiplegic limbs are evaluated, and the muscle strength recovery of patients is evaluated after rehabilitation training for 2 mo. The results show that after rehabilitation training, the muscle strength of hemiplegic patients with cerebral infarction is obviously restored, and the motor function of hemiplegic limbs and the ability of daily activities are significantly improved. Therefore, it can be concluded that rehabilitation training is conducive to promoting the recovery of limb motor function in patients with cerebral infarction hemiplegia and improving the life ability of patients, which has the promotion value. In the future research, large sample, multi-center randomized clinical control trial should be carried out to provide more reliable and effective rehabilitation mode for limb recovery of patients with cerebral infarction hemiplegia.
\end{abstract}

Key words: Cerebral infarction, rehabilitation training, hemiplegia, limb recovery

Cerebrovascular disease (CVD) is a common disease worldwide, which often occurs in the elderly. Cerebral infarction accounts for about $75 \%$ of CVD, which are characterized by high morbidity, mortality and disability, among which the incidence of hemiplegia caused by cerebral infarction is $80 \%{ }^{[1]}$. Hemiplegia refers to the paralysis of the upper and lower limbs on the same side, which is one of the main symptoms and sequelae of acute CVD and seriously affects the functional recovery of patients. Studies have reported that within $1 \mathrm{w}$ after cerebral infarction, about $45 \%$ of patients can't sit or stand normally, $75 \%$ of patients have difficulty in walking, and $75 \% \sim 8 \%$ of patients may occur limb paralysis ${ }^{[2,3]}$. Hemiplegia caused by acute cerebral infarction belongs to upper motor neuron injury, which mainly manifests as spasm, muscle weakness and impaired control and coordination of various active movements. The quality of life of stroke patients mainly depends on the degree of motor function recovery of hemiplegic limbs.
The rehabilitation of hemiplegia depends on the recovery process of cerebral tissue and cerebrovascular lesions and the plasticity of central nervous system. Functional training is the main means of function reorganization of central nervous system, and its training content includes the recovery of limb function on the affected side and the compensation of limb function on the uninjured side ${ }^{[4]}$. Studies have found that cerebral cortex has ipsilateral innervation, and functional training of healthy limbs can accelerate the establishment of ipsilateral cerebral collateral circulation and promote the reorganization of surrounding tissues. The simultaneous functional training of both limbs can promote the release of interhemispheric inhibition and promote the recovery of limb function of the affected side ${ }^{[5-7]}$.

Functional exercise is a long-term work, and patients need to adhere to exercise. Therefore, in order to enable stroke patients with hemiplegia to actively participate in rehabilitation training and rebuild confidence in life, functional training often starts from the acquisition of

*Address for correspondence

E-mail: malizhong_tzhzp@126.com 
the most basic ability of daily life activities. Daily life activities refer to a series of basic, common and repeated daily movements that people must master in order to live independently, such as eating, dressing and going to the toilet. Due to various motor dysfunction caused by hemiplegia, the daily activities of stroke patients are seriously affected, and most patients can't take care of themselves. The level of daily activity ability is an important influencing factor for the rehabilitation effect of hemiplegic patients and their return to society ${ }^{[8]}$. After the occurrence of hemiplegia of CVD, systematic rehabilitation intervention should be carried out to the patients as soon as possible according to their specific conditions. Many foreign studies and attempts have been made in this regard, and standardized treatment has been gradually formed in clinical practice, but its efficacy remains to be further studied and verified ${ }^{[9]}$. Therefore, in this study, it aims to explore the effect of rehabilitation training on limb recovery of patients with cerebral infarction hemiplegia, as well as the improvement of patient's ability of daily activities, so as to provide a theoretical basis for clinical rehabilitation training on limb recovery of cerebral infarction.

\section{MATERIALS AND METHODS}

\section{General information:}

46 patients with cerebral infarction hemiplegia hospitalized in the department of neurology and rehabilitation of Taizhou Hospital of Zhejiang Province from March 2014 to September 2018 are selected. All cases are diagnosed in accordance with the diagnostic criteria revised by the fourth academic conference on CVD in 1995, and are confirmed as ischemic stroke by head CT (computed tomography) or MRI (magnetic resonance imaging). The informed consent signed by the patients or their family members was obtained and this study was approved by the medical ethics committee of Taizhou Hospital of Zhejiang Province.

Inclusion criteria: Aged 45 to 65 y old; first onset with hemiplegia; clear consciousness; those who actively cooperate with rehabilitation training. Exclusion criteria: patients with hemiplegia caused by traumatic brain injury; patients with severe cognitive impairment and those who unable to understand and cooperate with rehabilitation training; and patients with severe heart, liver, lung, and kidney damage.

\section{Rehabilitation training method:}

First, exercise therapy. The specific exercise method is used to restore the patient's motor function. The focus of the rehabilitation of motor function in stroke patients with hemiplegia is to improve the patient's motor control ability. In the rehabilitation process of stroke patients with hemiplegia, the main functional training includes 7 items, as shown in fig. 1.

Placement of antispasmodic position: The typical spasm pattern in stroke patients with hemiplegia is spasm of the flexor muscles of the upper limbs and spasm of the extensor muscles of the lower limbs. Specific manifestations include adduction and rotation of shoulder joint, elbow joint flexion with forearm pronation, wrist joint flexion, thumb flexion adduction, extension, adduction, and pronation of hip joint, knee extension, plantar flexor varus, and flexion adduction of the toe. In the early stage of functional training, by properly placing the limbs of the patient in different

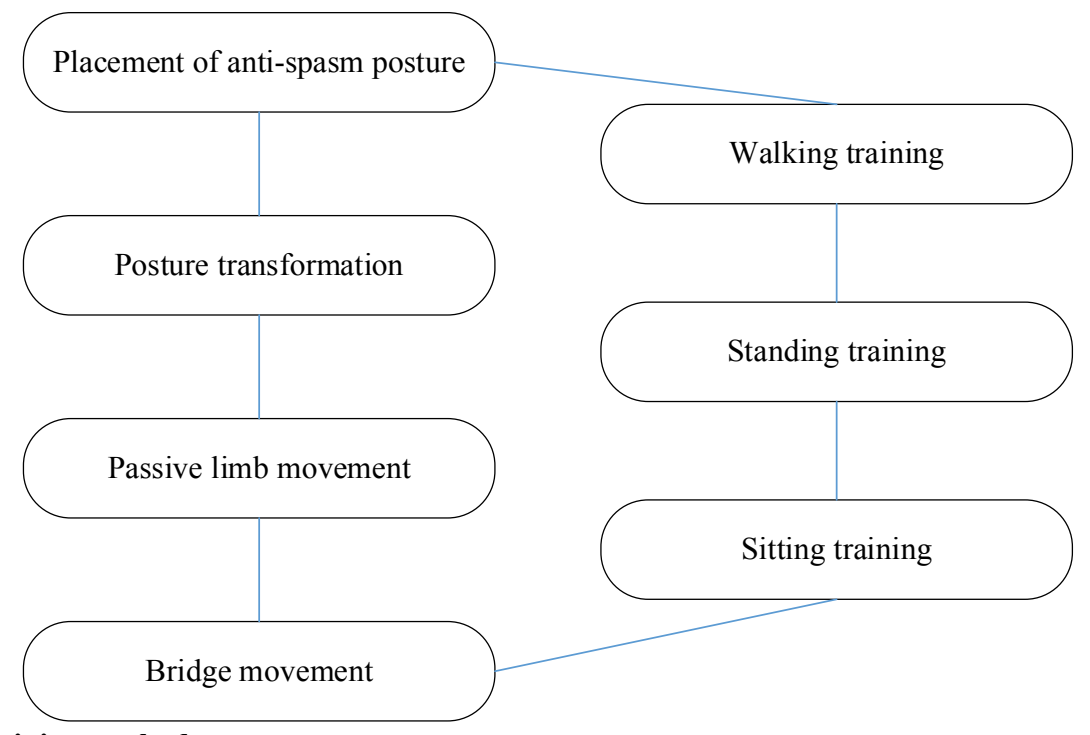


positions such as the supine position, the healthy lateral position, and the affected lateral position, the purpose that prevents spasm to appear or alleviate its degree is reduced.

Position transformation: Maintaining the same position for a long time cannot only cause complications such as bedsores and hypostatic pneumonia, but also promote the appearance of spasm by strengthening muscle advantages. For example, the supine position can strengthen the advantage of extensor muscle, the healthy lateral position can strengthen the flexor muscle advantage of the affected side, and the affected lateral position can strengthen the extensor muscle advantage of the affected side.

Passive movement of the limb: During the period of soft paralysis, the limb on the affected side has no voluntary movement, and the passive movement of the limb can not only prevent the limitation of joint movement, but also promote the blood circulation of the limb and increase the correct motion sensory input to affect the output. According to the motion range of the limb joint on the healthy side, the affected side should be moved from the proximal end of the limb to the distal end. The movement should be gentle and slow.

Bridge exercise: By increasing the strength of the lumbar dorsal muscle group and stretching the hip joint, patients can prepare for the transition from lying position to standing position. The completion of this exercise also determines whether patients can return to normal gait, including double bridge exercise and single bridge exercise. Double-bridge exercise refers to the patient taking the supine position, flexing the legs, lifting the buttocks and maintaining it for a while and letting go. When the patient can easily complete the double bridge exercise, the patient is asked to hang the healthy side leg, only the side leg flexes, and the hips are lifted, which is the single bridge movement.

Sitting training: It is the basis for standing, walking and performing some daily activities, starting at the same time as the above exercises, including sitting balance and endurance training. In order to avoid the semisitting position on the bed to prevent the strengthening of the extensor muscles of the lower extremities, training is carried out at the bedside or on the chair and the patient's feet should be stepped on the ground to prevent foot varus.

Station training and standing training: It should remind the patient to avoid maintaining the standing position or standing up only with the support of the lower limbs on the healthy side.

Walking training: It is started when the lower limbs of the affected side are heavier than half of the body weight and the patient can move forward. Note that the amount of early activity should not be too large. Walking aids may be used when appropriate, or family members may provide a small amount of support.

Second, Occupational therapy: Occupational therapy refers to the purposeful selection of some work activities according to the functional disorder or disability degree of patients, and to perform the treatment and training to maximize the recovery or improvement of living and working ability. When the patient is able to take a sitting position, it begins and it includes daily activities training such as eating, personal hygiene, dressing, etc.

In order to avoid discomfort of patient, functional training should be carried out $1 \mathrm{~h}$ after meals, 2 times/d, and $30 \mathrm{~min}$ each time. The training should be gradual, and the time and intensity should be adjusted according to the patient's movement, so as not to cause excessive fatigue or increase the degree of systemic spasm.

\section{Evaluation criteria for effects:}

The recovery of muscle strength is evaluated by the Lovett 6-level scoring method, which is divided into 6 levels of $0,1,2,3,4$, and 5. Level 0 represents a slight contraction of muscles that does not drive joint activity, grade 5 is normal muscle strength, which can resist adequate resistance and gravity. The higher the score, the better the muscle strength ${ }^{[10]}$. The evaluation time is before rehabilitation training and 2 mo after rehabilitation training.

Simple Fugl-Meyer motor function score is used for limb motor function in hemiplegic side ${ }^{[11-13]}$. According to the recovery process of hemiplegia, it is divided into five items: reappear reflex, voluntary movement which is completely manifested by common movement, voluntary movement which is partially separated from common movement, voluntary movement which is completely or highly separated from common movement and reflex to return to normal, and ataxia movement and balance. The total score is 100 points, including 66 points for upper limbs and 34 points for lower limbs. Each part of the scoring standard is divided into three levels, 0 points for complete non-execution, 1 for partial execution, and 2 for full execution. The higher the score, better the recovery of the motor function. The evaluation time is before rehabilitation 
training, $2 \mathrm{w}$ after rehabilitation training and $4 \mathrm{w}$ after rehabilitation training.

The ability of daily living activities is assessed by the British Standardized Barthel Index ${ }^{[14,15]}$. It includes 10 items: eating, bathing, grooming, dressing, going to the toilet, defecating, bed and chair shifting, flat moving and going up stairs. According to whether the patient needs help and its degree, it is divided into 3 points, 2 points, 1 point and 0 points, with a full score of 20 points. The higher the score, the stronger the ability of daily life activities. The evaluation time is before rehabilitation training, $2 \mathrm{w}$ after rehabilitation training and $4 \mathrm{w}$ after rehabilitation training.

\section{RESULTS}

Hemiplegia refers to the paralysis of the upper and lower limbs on the same side. As the most common functional disorder in stroke patients, hemiplegia is mainly manifested as incomplete or complete loss of voluntary movement of the limbs on one side, which is caused by damage of the pyramidal tract on one side, often accompanied by damage of the extrapyramidal system. Hemiplegia caused by cerebral apoplexy belongs to upper motor neuron injury, which mainly manifests as spasm, muscle weakness and impaired control and coordination of various active movements. The recovery mainly depends on the recovery process of brain tissue and vascular lesions, including the establishment of collateral circulation, edema regression around the lesion and hematoma absorption. In this study, it is found that before rehabilitation training, there are 9 patients with grade $0-1$ of upper limb muscle strength, 32 patients with grade 2-3, and 5 patients with grade 4-5. And there are 12 patients with grade $0-1$ of lower limb muscle strength, 30 patients with grade 2-3, and 4 patients with grade 4-5. After rehabilitation training for $2 \mathrm{mo}$, there are 0 patients with grade 0-1 of upper limb muscle strength, 18 patients with grade 2-3, and 28 patients with grade 4-5. And there are 0 patients with grade $0-1$ of lower limb muscle strength, 21 patients with grade $2-3$, and 25 patients with grade $4-5$, as shown in fig. 2A and fig. $2 \mathrm{~B}$.

Simplified Fugl-Meyer motor function is often used in limb function assessment. Barthel index is used to evaluate the ability of daily living activities. The above scale has good reliability and validity after being proved by Chinese experts and applied in China. Early limb rehabilitation training can stimulate part of brain cells to produce functional compensation, so that the nervous system can establish new links as soon as possible, thus promoting the recovery of limb function as soon as possible, and in the early stage of disease, limb function has a natural recovery trend, which is the best time for functional training. In this study, it is found that the motor function scores of patients before rehabilitation training, $2 \mathrm{w}$ after rehabilitation training and $4 \mathrm{w}$ after rehabilitation training are $20.45 \pm 3.23,60.42 \pm 8.25$, and $79.86 \pm 8.04$, respectively. The motor function scores increase significantly $2 \mathrm{w}$ after rehabilitation training and $4 \mathrm{w}$ after rehabilitation training, and the difference is statistically significant $(\mathrm{p}<0.05)$. The Barthel index of patients before rehabilitation training, $2 \mathrm{w}$ after rehabilitation training and $4 \mathrm{w}$ after rehabilitation training are $3.77 \pm 2.06,10.42 \pm 1.55$, and $15.35 \pm 1.78$, respectively. The score of daily life activity ability increases significantly after rehabilitation training for $2 \mathrm{w}$ and $4 \mathrm{w}$, and the difference is statistically significant $(\mathrm{p}<0.05)$, as shown in Table 1.

Cerebral infarction is a cerebrovascular clinical event caused by various causes of acute onset, rapid localized or diffuse brain function loss ${ }^{[16]}$. Hemiplegia refers to

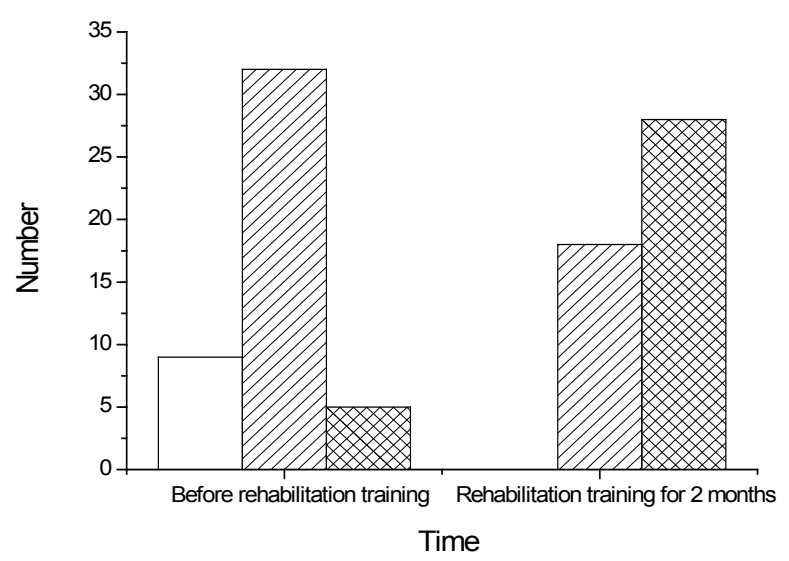

Fig. 2A: Recovery of muscle strength of the upper limbs Level 0-1 (a) Level 2-3 ( $(\aleph)$

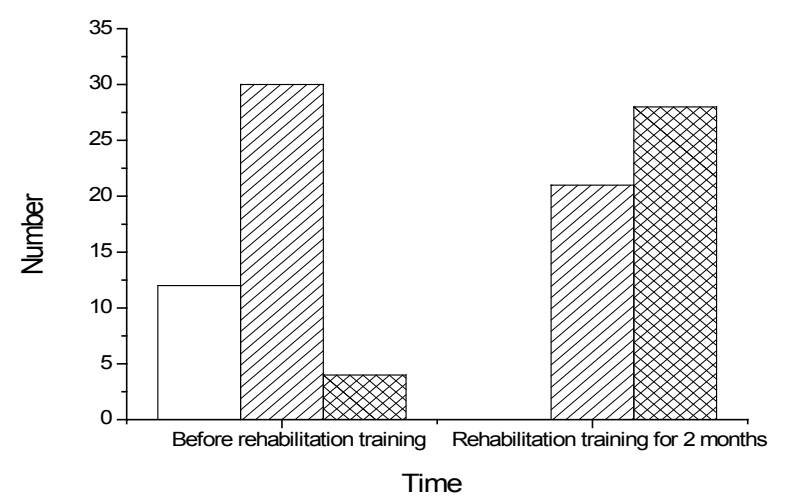

Fig. 2B: Recovery of muscle strength of the lower limbs Level $0-1$

$(\& \&)$ 
TABLE 1: SCORES OF PATIENTS MOTOR FUNCTION AND RESULTS OF BARTHEL INDEX

\begin{tabular}{lcc}
\hline Time & $\begin{array}{c}\text { Motor function } \\
\text { score }\end{array}$ & $\begin{array}{c}\text { Barthel } \\
\text { index }\end{array}$ \\
\hline $\begin{array}{l}\text { Before rehabilitation training } \\
\text { Rehabilitation training for }\end{array}$ & $20.45 \pm 3.23$ & $3.77 \pm 2.06$ \\
$2 \mathrm{w}$ & $60.42 \pm 8.25$ & $10.42 \pm 1.55$ \\
$\begin{array}{l}\text { Rehabilitation training for } \\
4 \mathrm{w}\end{array}$ & $79.86 \pm 8.04$ & $15.35 \pm 1.78$ \\
\hline
\end{tabular}

TABLE 2: COMPARISON OF OVULATION BETWEEN THE TWO GROUPS $(\mathrm{N}=41)$

\begin{tabular}{lcc}
\hline Group & $\begin{array}{c}\text { Number of } \\
\text { mature follicles }\end{array}$ & $\begin{array}{c}\text { Endometrial thickness } \\
\text { on HCG days }(\mathrm{cm})\end{array}$ \\
\hline Experimental & $1.20 \pm 0.45$ & $0.92 \pm 0.23$ \\
group & $1.08 \pm 0.35$ & $0.80 \pm 0.21$ \\
Control group & 2.04 & 1.01 \\
$t$ & $>0.05$ & $<0.05$ \\
$\mathrm{p}$ &
\end{tabular}

the paralysis of the upper and lower limbs on the same side, which is caused by the damage of the pyramidal tract on one side, and is often accompanied by the damage of the extrapyramidal system. It is characterized by incomplete movement or complete loss of one limb, which is the main symptom of acute CVD. The quality of life of stroke patients mainly depends on the degree of motor function recovery of hemiplegic limbs. Therefore, how to promote the recovery of motor function in hemiplegic patients, improve self-care ability to the greatest extent and improve the quality of life has become the focus of current research.

For the recovery of hemiplegia, in addition to the active rescue of injured brain cells, promote the recovery of the pathological process and the formation of collateral circulation, but also give full play to the central nervous system function reorganization. For the rehabilitation of hemiplegia, in addition to actively rescue the damaged brain cells, promote the recovery of pathological processes and the formation of collateral circulation, it is necessary to give full play to the functional reorganization of the central nervous system. Functional training is the main condition for the functional reorganization of the central nervous system. By strengthening the strength training of weak muscles and inhibiting abnormal and original reflex activities, the motor pattern can be improved and the normal motor pattern can be reconstructed. Limb rehabilitation training can stimulate part of brain cells to produce functional compensation, so that the nervous system can establish new links as soon as possible, thus promoting the recovery of limb function as soon as possible and improving the ability of patients to take care of themselves and the quality of life.

The results of this study show that after rehabilitation training, the limb recovery effect of patients with cerebral infarction hemiplegia is significant, and the patient's motor function and daily life activity ability are significantly improved compared with those before rehabilitation training. However, the sample size of this study is relatively small, and the sample size should be increased in later similar studies and a multicenter, multi-group randomized control study should be conducted. In addition, in the future rehabilitation training for patients, the therapeutic staff should strictly adjust the plan according to the evaluation results, and develop individualized training programs for patients.

\section{Author's contributions:}

Lizhong Ma and Qilong Deng contributed equally to this work.

\section{Acknowledgements:}

This project was supported by the National Natural Science Foundation of China (Grant No. 81804185).

\section{Conflict of interests:}

The authors declared no conflict of interest.

\section{REFERENCES}

1. Chu H, Zhang S, Fu J, Dong H, Guo R, Zhao G, et al. TIE's flying acupuncture for acute cerebral infarction hemiplegia: a randomized controlled trial. Zhongguo Zhen Jiu 2017;37(11):1153-6.

2. Min GU, Si-Wei LI, Bao-Jin LI, Cheng LI, Yun QU. Effect of motor relearning programme on motor function recovery of acute stroke patients with hemiplegia. Chin J Contemp Neurol Neurosurg 2017;17(3):197-201.

3. Kim WS, Kim MJ. Individual joint contribution to body weight support in the affected lower limb during walking in post-stroke hemiplegia. Top Stroke Rehabil 2017;24(3):170-6.

4. Lingyan HE, Feng L, ChenZ. Observation of Electromyographic biofeedback stimulation on acupoints to improve foot drop in stroke patients with hemiplegia. Rehabil Med 2017;27(1):40.

5. Sun R, Tian L, Fang X, Du X, Zhu B, Song Z, et al. Clinical study of post-stroke upper limb spasmodic hemiplegia treated with jingou diaoyu needling technique and Bobath therapy. Zhongguo Zhen Jiu 2017;37(4):372-6.

6. Goto H, Tanaka T, Suetsugi N, Kubotsu Y, Momozaki N, Mizuta T. Non-cirrhotic Portal-systemic Encephalopathy with total aphasia and right hemiplegia following transient right hemiparesis: A case report. Brain nerve 2018;70(2):155-9.

7. Shin HE, Suh HC, Kang SH, Seo KM, Kim DK, Shin HW. Diagnostic challenge of diffusion tensor imaging in a patient 
with hemiplegia after traumatic brain injury. Ann Rehabil Med 2017;41(1):153-7.

8. Marquez-Chin C, Bagher S, Zivanovic V, Popovic MR. Functional electrical stimulation therapy for severe hemiplegia: Randomized control trial revisited. Can J Occup Ther 2017;84(2):87-97.

9. Bidaki R, Zarepur E. Intermittent hemiplegia in a boy with primary moyamoya disease: A case report from Iran. Iran J Child Neurol 2017;11(2):65-8.

10. Han L, Li SG, Zhai HW, Guo PF, Chen W. Effects of weight training time on bone mineral density of patients with secondary osteoporosis after hemiplegia. Exp Ther Med 2017;13(3):961-5.

11. Saita K, Morishita T, Hyakutake K, Fukuda H, Shiota E, Sankai Y, et al. Combined therapy using botulinum toxin A and single-joint hybrid assistive limb for upper-limb disability due to spastic hemiplegia. J Neurol Sci 2017;373:182-7.

12. Donepudi B, Trottier S. A seizure and hemiplegia following contrast exposure: understanding contrast-induced encephalopathy. Case Rep Med 2018:1-4.

13. Giacanelli M, Petrucci A, Lispi L, Luna R, Neri G, Gurrieri F, et al. ATP1A3 mutant patient with alternating hemiplegia of childhood and brain spectroscopic abnormalities. J Neurol Sci 2017;379:36-8.

14. Ito $\mathrm{T}$, Narugami $\mathrm{M}$, Egawa $\mathrm{K}$, Yamamoto $\mathrm{H}$, Asahina $\mathrm{N}$, Kohsaka S, et al. Long-term follow up of an adult with alternating hemiplegia of childhood and a p. Gly755Ser mutation in the ATP1A3 gene. Brain Dev 2018;40(3):226-8.

15. Shah I, Nandy S, Kumari R. Hemiplegia as a presentation of HIV infection in children: A report of 3 cases. J Family Med Prim Care 2017;6(2):439-41.

16. Zheng $\mathrm{Y}$, Song $\mathrm{T}$, Zhang LL, Wei N. Immunomodulatory effects of $\mathrm{T}$ helper 17 cells and regulatory $\mathrm{T}$ cells on cerebral ischemia. J Biol Regul Homeost Agents 2018;32(1):29-35.

This is an open access article distributed under the terms of the Creative Commons Attribution-NonCommercial-ShareAlike 3.0 License, which allows others to remix, tweak, and build upon the work non-commercially, as long as the author is credited and the new creations are licensed under the identical terms

This article was originally published in a special issue, "Trends in Therapeutic Management of Various Clinical Conditions II" Indian J Pharm Sci 2021:83(2)Spl issue;30-35 